\title{
Improved HSI Color Space for Color Image Segmentation
}

\author{
Rodolfo Alvarado-Cervantes and Edgardo M. Felipe-Riveron* \\ Center for Computing Research, National Polytechnic Institute, Juan de Dios Batiz w/n, Col. \\ Nueva Industrial Vallejo, P.O. 07738, Mexico \\ ateramex@gmail.com, edgardo@cic.ipn.mx
}

\begin{abstract}
We present an interactive, semiautomatic image segmentation method that processes the color information of each pixel as a unit, thus avoiding color information scattering. The color information of every pixel is integrated in the segmented image by an adaptive color similarity function designed for direct color comparisons. The border between the achromatic and chromatic zones in the HSI color model has been transformed in order to improve the quality of the pixels segmentation when their colors are very obscure and very clear. The color integrating technique is direct, simple and computationally inexpensive, and it has also good performance in low chromaticity and low contrast images. It is shown that segmentation accuracy is above $95 \%$ as average and that the method is fast. These results are significant when compared to other solutions found in the current literature.
\end{abstract}

Keywords: Color image segmentation, Adaptive color similarity function, Improved HSI color model, Achromatic zone definition.

\section{Introduction}

At present, several segmentation techniques are available for color images, a good amount of them are monochromatic methods applied on the individual planes in different color spaces where the results are combined later in different ways [3]. A common problem to this approach is that when the color components of a particular pixel are processed separately the color information is so scattered in its components that most of the color information is lost [1] [3] [4].

In this work, an interactive, semiautomatic image segmentation method is presented that uses the color information for each pixel as a whole, thus avoiding color information scattering.

One weakness in the characterization of the achromatic region as presented in [5], [7] and [10] is its poor performance in the border regions of achromatic and chromatic zones, both in low and high brightness, due to the fact that the commonly used HSI color model does not take into account the human visual response at low and high brightness. Human vision has a nonlinear perceptual response to luminance [11]. This

* Corresponding author. 
problem was overcome by modifying the saturation value of every pixel by a factor that reflects the human exponential response to brightness. We show this improvement in Section 3 comparing the achromatic zone obtained by both methods.

The results tabulated in Section 3 show that the segmentation method presented in this paper offers a useful and efficient alternative for the segmentation of objects with different colors in relatively complex color images with good performance in the presence of the unavoidable additive noise. It has also good performance in gray level and low contrast images [5].

\section{Description of the Method}

The segmentation method proposed in this paper relies on the calculation of a color similarity function for every pixel in a RGB 24-bit true color image to form what we call a Color Similarity Image (CSI), which is a gray level image. The color similarity function allows the clustering of the many thousands of colors representing the same perceived color in a single gray output image. The CSI is then automatically thresholded and the output can be used as a segmentation layer, or it can be modified with morphological operators to introduce geometric enhancements if they are needed. The generation of a CSI only requires calculating Eq. 1 (below) for every pixel in the RGB input image. Thus the complexity is linear with respect to the number of pixels of the source image and for that reason inexpensive computationally.

Firstly, we compute the color centroid and color standard deviation of a small sample consisting of few pixels (less than 3-4 pixels per color). The computed centroid represents the desired color to be segmented using the technique we designed for that purpose.

Then, our color similarity function uses the color standard deviation calculated from the pixel sample to adapt the level of color scattering in the comparisons. The result of a particular similarity function calculation for every pixel and the color centroid (meaning the similarity measure between the pixel and the representative color value) generates the CSI.

The CSI can be thresholded with any automatic thresholding method. To obtain the results presented in this work we used Otsu's method [8] [9].

To obtain the CSI we calculate for every pixel $(i, j)$ in the image the following color similarity function (Eq. 1).

$$
S_{i, j}=e^{\left(\frac{-\Delta_{h}{ }^{2}}{2 \sigma_{h}{ }^{2}}\right)} * e^{\left(\frac{-\Delta_{s}{ }^{2}}{2 \sigma_{s}{ }^{2}}\right)} * e^{\left(\frac{-\Delta_{i}{ }^{2}}{2 \sigma_{i}{ }^{2}}\right)}
$$

where $\Delta_{h}$ is the hue distance between hue $(i, j)$ and the average_hue; $\Delta_{s}$ is the saturation distance between saturation $(i, j)$ and the average_saturation; $\Delta_{i}$ is the intensity distance between intensity()and the average_intensity; $\sigma_{h}$ is the hue standard deviation of the sample; $\sigma_{s}$ is the saturation standard deviation of the sample; $\sigma_{i}$ is the intensity standard deviation of the sample. In Equation (1) the color information is integrated giving high importance to perceptual small changes in hue, as well as 
giving wide or narrow tolerance to the intensity and saturation values depending on the initial sample, which is representative to the desired color to be segmented.

The common disadvantages attributed to the HSI color space such as the irremovable singularities of hue in very low saturations or the periodical nature of hue [7], which is lost in its standard representation as an, are overcome in our technique using vector representation in $\mathfrak{R}^{2}$, in the separation of chromatic and achromatic regions, and in the definition of the $\Delta_{h}, \Delta_{s}$ and $\Delta_{i}$ distances.

\subsection{Pixel Sample Selection}

The pixel sample is a representation of the desired color(s) to be segmented from a color image. From this pixel sample we calculate two values to feed our segmentation algorithm: the color centroid and a measure of the dispersion from this centroid, in our case the standard deviation, they can be consulted for reference in [5] and [10].

\subsection{The Achromatic Zone G}

The achromatic zone G is the region in the HSI color space where no hue is perceived by the human. This means that color is perceived only as a gray level because the color saturation is very low or the intensity is either too low or too high.

In order to model better the human visual response in the abrupt corners regions near the union zones of the two cones with the cylinder of the singularity zone $G$, as presented in [5], [7] and [10], we found convenient to modify the characterization of the HSI color model for colors belonging to regions with very low or very high brightness. This step will permit us to differentiate the colors in these extreme regions, because the human response to brightness does not have this possibility. In order to adjust the zone $\mathrm{G}$ to a better model nearer to the human response to brightness we introduced an exponential function with three parameters to define the improved singularity of zone G: saturation threshold (st), inflection point (ip), and Slope (Fig 1). Then, the saturation image will be affected now by a factor calculated from the inflection point (ip), and Slope for every pixel (P) as shown in Eq.(2):

$$
N e w_{-} \text {Saturation_ }(P)=\left(1-\text { Slope } *(e)^{\text {abs }(i p-\text { int ensity }(P))}\right) * S_{\text {Saturation }}(P)
$$

where Slope is a parameter representing the degree of affectation of the exponential factor to the saturation attribute for every pixel; abs( ) represents the absolute value function and intensity( ) is defined as an average of the RGB channels in Eq. (6) in [5]. After calculating the New_Saturation values for the source image, it is thresholded with the saturation threshold (st) to obtain the effects of the improved achromatic zone $\mathrm{G}$.

We found good performance with the parameters empirically determined in the following ranges: st $\epsilon[0.07,0.1]$, ip $\epsilon[0.5,0.6]$, and Slope $\epsilon[1,1.5]$.

The statistical values needed in Eq. (1) are calculated from the pixel sample using the common statistics formulae adapted to our model. The details about all this can be consulted in [5] and [10]. 


\section{Experiments and Results}

In this section we describe the experiments and present the results achieved from our segmentation method applied to two classical color images in RGB 24-bit true color format. These experiments consisted of segmenting color regions according to the following two steps:

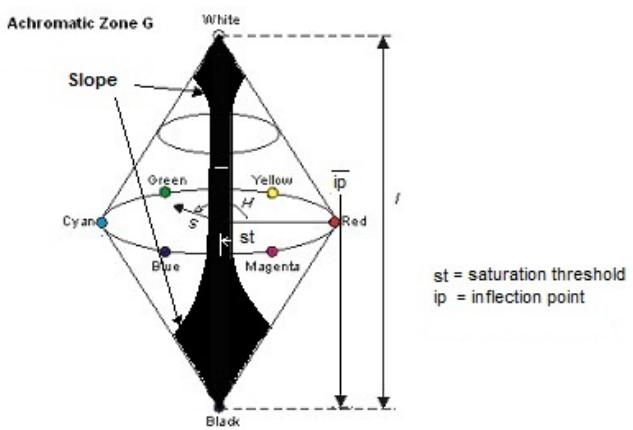

Fig. 1. The new achromatic zone $G$

Selection of the pixel sample. This is the only step to be left up to the user, and [5] can be consulted as a guide. This step is automatic; its output is a gray image showing the similarity of each pixel of the RGB true color image to the color centroid formed with the chosen pixel sample taken from of the region of interest to be segmented, being white for $100 \%$ of similarity and black for $0 \%$.

The user can now threshold the CSI using, for example, the non-supervised Otsu thresholding method [8], [9] as in this case.

We can also separate the achromatic area to obtain a better segmentation. This achromatic zone can be subdivided later using only intensity as a discriminative characteristic. In figures 2, 3 and Table 1 we show the improved results of our new definition of $\mathrm{G}$ in Eq. (2) with respect to the former results presented in [5], [7] and [10].

In all the composite images we used the XOR logical function to avoid the possibility that one pixel could belong simultaneously to two different color-segmented zones or regions.

Next, we demonstrate the effectiveness of the proposed color segmentation method in some relatively complex color images.

Figure 2 (left) shows the results of the application of our method to the RGB color image (sized $200 \times 200$ pixels and with 33753 different colors) of the popular baboon image. In this image we can see four main hues of colors despite the many thousands of actual RGB values to represent them: The red part of the baboon's nose, the blue part of the nose, the orange eyes and the yellow-orange part of the fur.

In our experiments we do not use any preprocessing at all. We obtained $95.5 \%$ of pixels segmented properly (See Table 1). In figure 2 we show the results with the old (center) and new achromatic region $\mathrm{G}$ (right) for appreciation. The improvement in quality is significant. 

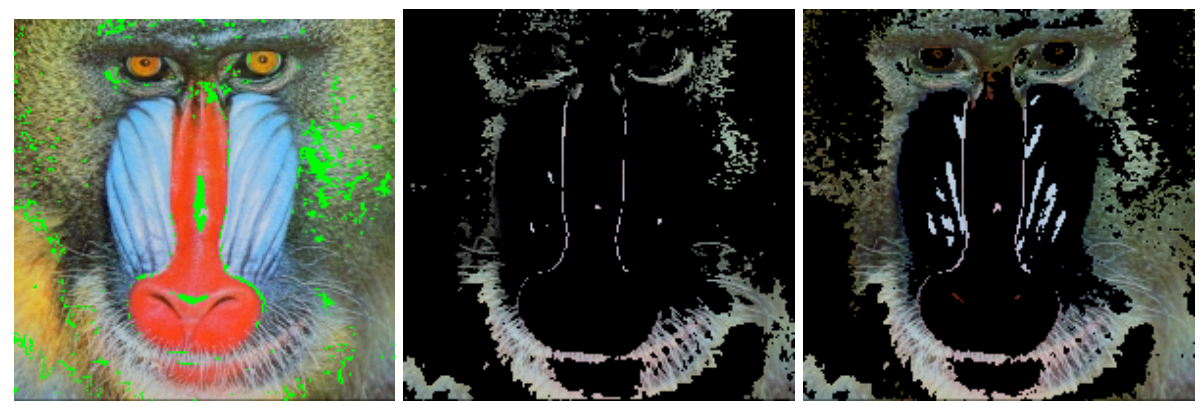

Fig. 2. Composite image of 5 segmented colors (left) and results obtained with the old (center) and the new achromatic zone $\mathrm{G}$ (right)

In order to evaluate the efficiency of the color segmentation method and due to the difficulty of obtaining a ground-truth for each complex image to which we applied the method or to compare the results from different methods, the evaluation was based on the number of pixels not segmented with respect to the total number of pixels in the corresponding image. This ratio combines the pixels not belonging to any color cluster and those selected by two or more clusters (obtained by means of the XOR operation) with respect to the total number of pixels in the image It gives us a measure of the segmentation efficiency.. In general the number of selected pixels for the samples depends on the complexity of the image (texture of objects), on the diversity of colors and on their spatial distribution in the image. As it can be observed from Table 1, the average accuracy of the color segmentation in both complex images, without an exhaustive selection of colors and a few numbers of pixels (4-5 as average) per color sample, is $95.91 \%$.

In Figure 3 (left) a composite image of 5 segmented colors (with the nonsegmented pixels in pseudocolor) of a color fabric is shown; it contains an achromatic region with high saturation but low brightness that is very difficult to separate with the former $\mathrm{G}$ zone (Figure 3 center) but easily separated with the new one shown in Fig. 3 (right). Figure 3 shows the original and a composite with the non-segmented pixels in pseudocolor. We obtained $95.3 \%$ of pixels segmented.
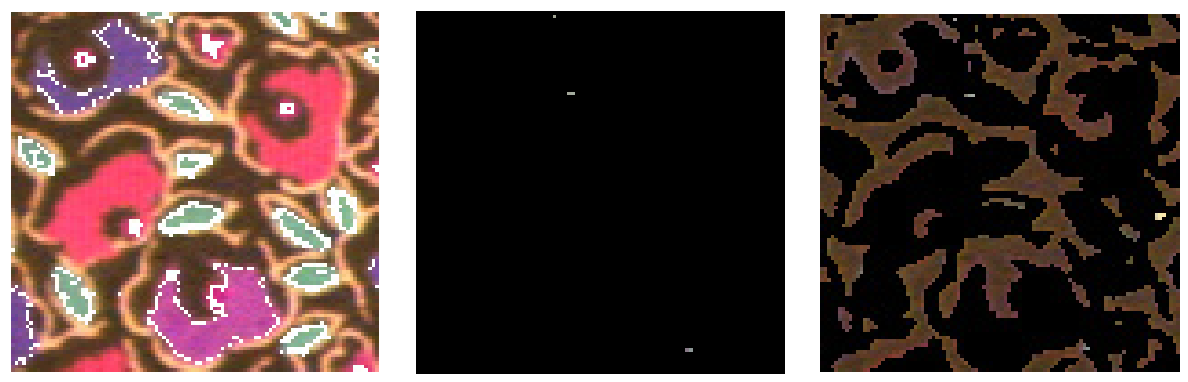

Fig. 3. Composite image of 5 segmented colors (left) and results obtained with the old (center) and the new achromatic zone $\mathrm{G}$ (right) 
When we compare the new achromatic region $G$ with the former one, the difference is astonishing whereas the new achromatic area seems correctly segmented the former one segmented only a couple of pixels.

Table 1 show the results obtained in the segmentation of two images. The last column of Table 1 shows the percent of segmented pixels obtained in these images.. In all cases, the possibility that one pixel could belong to two different colors segmented zones or regions have been avoided by means of the application of the XOR logical function (of two or more partial segmentations). A result with many black pixels indicates that has been coincidence in segmented pixels from two or more partial segmentations considered in the XOR operation.

As it can be observed from Table 1, the average accuracy of the color segmentation in both complex images, without an exhaustive selection of colors and a few numbers of pixels (4-5 as average) per color sample, is $95.4 \%$.

Table 1. Results of the global segmentation per image

\begin{tabular}{|c|l|c|c|c|c|c|c|}
\hline No. & Image & $\begin{array}{c}\text { Number } \\
\text { of pixels } \\
\text { in image }\end{array}$ & $\begin{array}{c}\text { Number } \\
\text { of colors } \\
\text { (levels) }\end{array}$ & $\begin{array}{c}\text { Number } \\
\text { of colors } \\
\text { (levels) } \\
\text { selected }\end{array}$ & $\begin{array}{c}\text { Total } \\
\text { number } \\
\text { of pixels } \\
\text { used as } \\
\text { samples }\end{array}$ & $\begin{array}{c}\text { Number } \\
\text { of non- } \\
\text { segmented } \\
\text { pixels }\end{array}$ & $\begin{array}{c}\% \text { of } \\
\text { segmented } \\
\text { pixels }\end{array}$ \\
\hline 1 & Baboon & 40000 & 33753 & 5 & 31 & 1803 & 95.5 \\
\hline 2 & Fabric & 9900 & 9349 & 5 & 23 & 465 & 95.3 \\
\hline
\end{tabular}

\section{Conclusions}

The results in the previous section, demonstrate that the color segmentation method presented in this paper offers a useful and efficient alternative for the segmentation of objects with different colors in relatively complex color images with good performance in the presence of the unavoidable additive noise, and in images with low contrast, and with very low chromaticity.

The steps required to obtain a good segmentation of regions with different colors by using the proposed methodology are usually straightforward, simple and repetitive. If color is a discriminative characteristic in the layer of interest, only the selection of a given threshold to the color similarity image CSI is needed to obtain a good segmentation result. From many experiments we have observed that a good percentage of colors were segmented in a straightforward way only by thresholding the Color Similarity Image. The method discriminates whichever type of different color objects independently on their shapes and tonalities in a very straightforward way.

The new characterization of the achromatic region improves its performance, compared to that presented in [5], [7] and [10], due to the affectation of saturation by an exponential factor in an effort to better model the human visual response in case of very low or very high brightness. The improvement in quality of its results is significant and can be visually appreciated in examples of Section 3. 
One of the inconveniences of the proposed color segmentation method is the degree of human intervention, summarized in the necessity of selecting a pixel sample. This sample has to be carefully chosen in order to obtain the best results. An associated problem of the use of a pixel sample is to obtain the number of data points necessary to capture the color distribution. These related problems will be treated in future developments of the algorithm enforced to obtain a complete automatic version.

Acknowledgements. The authors of this paper wish to thank the Computing Research Center (CIC), Mexico; Research and Postgraduate Secretary (SIP), Mexico, and National Polytechnic Institute (IPN), Mexico, and CONACyT, Mexico, for their support to this work.

\section{References}

1. Alvarado-Cervantes, R.: Segmentación de patrones lineales topológicamente diferentes, mediante agrupamientos en el espacio de color HIS. M. Sc. Thesis, and Center for Computing Research, National Polytechnic Institute, Mexico (2006)

2. Felipe-Riveron, E.M., García-Ramos, M.E., Levachkine, S.P.: Problemas potenciales en la digitalización automática de los mapas cartográficos en colores. In: Proceedings of International Congress on Computation CIC IPN, Mexico City, Mexico (2000)

3. Cheng, H., Jiang, X., Sun, Y., Wang, J.: Color image segmentation: Advances and prospects. Pattern Recognition 34(12), 2259-2281 (2001)

4. Hanbury, A., Serra, J.A.: 3D-polar coordinate colour representation suitable for image analysis. Technical Report PRIP-TR-77, Austria (2002)

5. Alvarado-Cervantes, R., Felipe-Riveron, E.M.: An Adaptive Color Similarity Function for Color Image Segmentation. In: San Martin, C., Kim, S.-W. (eds.) CIARP 2011. LNCS, vol. 7042, pp. 113-124. Springer, Heidelberg (2011)

6. Gonzalez, R.C., Woods, R.E.: Digital Image Processing, 3rd edn. Prentice-Hall, USA (2008)

7. Plataniotis, K.N., Venetsanopoulos, A.N.: Color Image Processing and Applications, 1st edn. Springer, Germany (2000)

8. Otsu, N.: A Threshold Selection Method from Gray-Level Histograms. IEEE Transactions on Systems, Man and Cybernetics 9(1), 62-66 (1979)

9. Sezgin, M., Sankur, B.: Survey over image thresholding techniques and quantitative performance evaluation. Journal of Electronic Imaging 13(1), 146-165 (2003), doi: $10.1117 / 1.1631315$

10. Alvarado-Cervantes, R., Felipe-Riveron, E.M., Sanchez-Fernandez, L.P.: Color Image Segmentation by Means of a Similarity Function. In: Bloch, I., Cesar Jr., R.M. (eds.) CIARP 2010. LNCS, vol. 6419, pp. 319-328. Springer, Heidelberg (2010)

11. Poynton, C.: (2002), http: / / www . poynton.com/ PDFs / GammaFAQ .pdf

12. Dubey, A., Bhattacharya, I., Godbole, S.: A Cluster-Level Semi-supervision Model for Interactive Clustering. In: Balcázar, J.L., Bonchi, F., Gionis, A., Sebag, M. (eds.) ECML PKDD 2010, Part I. LNCS, vol. 6321, pp. 409-424. Springer, Heidelberg (2010) 